\title{
INTERPRETING DATA FROM POLARIZED PROTON BEAMS
}

\author{
G L KANL: and UP SUKHATME \\ Physics Depatment, Unuersity of Michugan, Ann Arbor, Michigan $48104^{*}$ \\ Recelved 5 Februıry 1974 \\ (Revised 8 April 1974)
}

\begin{abstract}
The reactions $p p \rightarrow p p$ and $p p \rightarrow \Delta^{++} n$ with polarrzed beam and/or polanzed target are currently under investigation at the Argonne ZCS We discuss how to interpret various measured quantities in terms of anplitudes whose behavior is familar (as functions of s.t) For $\mathrm{pp}$ total cross sections and elastic sedtering. Argonne measurements will yield $\operatorname{Im} \varphi_{2}(s, t=0)$ and the rather complicated combination $2\left|\varphi_{5}\right|^{2}+\operatorname{Re}\left(\varphi_{1} \varphi_{2}^{*}-\varphi_{3} \varphi_{4}^{*}\right)$, where $\varphi_{1}(a=1$, , 5) are conventiond s-channel helicity amplitudes the forward direction $(t=0)$ is of specid interest We find that for both pp $\rightarrow$ pp and pp $\rightarrow \Delta^{++} n$, polarized beam polarized target experiments plus the rather general (testable) assumptoon that amplitudes with the same s-channel helicity thip quantum numbers are proportsonal, are sufticient to fully determme all non-vanshing amplitudes at $t=0$ Numencal estimates of come observables, based on calculations in a specific model, are also given
\end{abstract}

\section{Introduction}

The scattering of high energy polarized proton beams (recently avalable at the Argonne Zero (iradient Synchrotron) on polarized proton targets, yields considerable new information about scattering amplitudes. In this article, we study (m terms of $s$-channel helicity amplitudes) what features of the amplitudes can be obtained from polarized beam-target experiments In particular, we give a detalled description of total cross sections, elastic scattering (sect 2) and the process $\mathrm{pp} \rightarrow \Delta^{++} \mathrm{n}$ (sect 3), since these experiments are currently in progress at Argonne

We have used $s$-channel helicity amplitudes (among the many equivalent choices) for three reasons, (1) they are the only set which embody the angular momentum conservation constraints in the forward direction in a simple way, without mixing amplitudes. (11) many particle physicists have developed an intuition about the behaviour of the "standard" amplitudes $\varphi_{1}, \quad, \varphi_{5}$ for NN elastic scattering as functions of $s, t$ and about their sizes, (111) if $s$-channel helicity conserving absorptıve rescattering effects are important in determining the structure of amplitudes, as is considered likely, then only the $s$-channel helisity amplitudes will have a simple physical interpretation for their $t$-dependence (e $g$ the pattern of zeros)

\footnotetext{
* Revearch supported un part by the US Atomic Inergy Commission
} 
We began this analysis mainly to satisfy our own curiosity about how to interpret the results of the ANL experiments Our attitude has not been to solve formal problems of what can in principle be measured (which has in any case been done $[1,2]$ for pp scattering), but to relate the observables to our conventional understanding of amplitudes For example, the total cross section difference $\sigma_{\mathrm{T}}(\uparrow \uparrow)-\sigma_{\mathrm{T}}(\uparrow \downarrow)$, defined in a precise manner below, is very simply given by $\operatorname{Im} \varphi_{2}(s, t=0) . \varphi_{2}$ is a double flip amplitude with net helicity flip zero It would vanish identically at $t=0$ for exchanges of any number of particles with definite parity, but a non-zero value can arise from particle exchange with absorptive rescattering The man particle exchanges are $\pi$, which is mainly real, and vector plus tensor $\left(\omega+f, \rho+A_{2}\right)$ which are supposed to add to give a real amplitude in the exotic pp channel Thus, at the simplest level $\operatorname{Im} \varphi_{2}(0)=0$, and a small non-7ero value gives us information at a rather detaled level, about such matters as phase rotation due to absorption corrections, cxchange degeneracy breaking, and the phase of $\pi$ exchange

Several such analyses corresponding to vanous observables are given below (sect 4) Furthermore, we find particularly interesting results in the forward direction $(t=0)$ At $t=0$ it turns out for both $\mathrm{pp} \rightarrow \mathrm{pp}$ and $\mathrm{pp} \rightarrow \Delta^{++} \mathrm{n}$ that the current experments with polarized beam and target, plus the rather general (testable) assumption that amplitudes with the same $s$-channel helicity flip quantum numbers ate proportional [3], allow a full determination of all the remaining non-vanıshing amplitudes

We begn with the formalism, and express the observables in terms of the $s$ channcl helicity amplitudes, $\varphi_{1}, . \quad, \varphi_{5}$ for $p p \rightarrow p p$ and a similarly defined set for $p p \rightarrow \Delta^{++} n$ General arguments are given where possible for the behavior of the amplitudes and observables Finally, numerical predictions are given for some observables based on calculations in a definite model [4]

\section{Elastic scattering and total cross sections}

We will work in the $\mathrm{c} m$ frame with a right-handed coordinate system $X Y Z$ fixed in space The proton beam travels along the positive $Z$-axis (horizontal) and the positive $Y$-axis points vertically upward

Since the Argonne ZGS has a vertical magnetic field, it is simplest to have incident heans with vertical polarization Beams with spin pointing along the direction of motion (helicity eigenstates) will be experimentally feasible in the near future In view of the state of technology for polarized beams and targets, we shall confine our discussion only to spins pointing along the $Y$ and $Z$ axes. (An extension to spins pointing along the $X$ axis is completely straightforward)

The single particle spin eigenstates of $S_{Y}$ will be denoted by $|\uparrow\rangle($ spin up) and

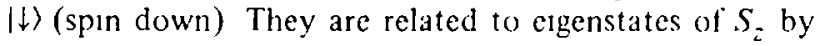




$$
|\uparrow\rangle=\frac{1}{\sqrt{2}}\left[\left|S_{z}=+\frac{1}{2} h\right\rangle+l\left|S_{z}=-\frac{1}{2} h\right\rangle\right], \quad|\downarrow\rangle=\frac{1}{\sqrt{2}}\left\{\left|S_{z}=+\frac{1}{2} h\right\rangle-l\left|S_{z}=-\frac{1}{2} h\right\rangle\right] .
$$

We wish to use the helicity eigenstates $|+\rangle$ and $\mid$.$) Noting that the target moves$ along the negative $Z$-axis in the $\mathrm{c} m$ frame, we readily obtain

$$
\begin{aligned}
& \left.\left.\right|_{\downarrow} ^{\uparrow}\right\rangle_{\text {beam }}=\frac{1}{\sqrt{2}}[|+\rangle \pm \imath|-\rangle]_{\text {beam }}, \\
& |\rangle_{\downarrow \text { target }}=\frac{1}{\sqrt{ } 2}[|-\rangle \pm \imath|+\rangle]_{\text {target }}
\end{aligned}
$$

Then the initial two-particle states which can be formed are (writing beam particle first)

$$
\begin{aligned}
& |\uparrow \uparrow\rangle=\frac{1}{2}[\imath|++\rangle+|+-\rangle-|-+\rangle+\imath|--\rangle],|\uparrow \downarrow\rangle=\frac{1}{2}[-t|++\rangle+|+--\rangle+|-+\rangle+t|--\rangle], \\
& |\downarrow \uparrow\rangle=\frac{1}{2}[t|++\rangle+|+-\rangle+|-+\rangle-l|\cdots-\rangle], \quad|\downarrow \downarrow\rangle=\frac{1}{2}[-t|++\rangle+|+--\rangle-|\cdots+\rangle-t|-\rangle]
\end{aligned}
$$

Proton-proton elastic scattering has five independent amplitudes Matrix elements for the reaction $(a+b \rightarrow c+d)$ between the helicity states $| \pm \pm\rangle$ are labeled in table 1 with conventional amplitude names $\varphi_{1}, \quad, \varphi_{5}$ [5] In the forward direction, conservation of $J_{2}$ requires that $\varphi_{4}(s, t=0)=\varphi_{5}(s, t=0)=0$, the other am. plitudes need not vanish at $t=0$

\section{Total cross sections}

We now express the total cross sections corresponding to various initial polari-

\begin{tabular}{|c|c|c|c|}
\hline & & $n$ & $x$ \\
\hline$\langle++|M|++\rangle$ & $=\varphi_{1}=\langle-|M|--\rangle$ & 0 & 0 \\
\hline$\langle++|M|+-\rangle$ & $=\varphi_{5}=-\{--|M|-+\rangle$ & 1 & 0 \\
\hline$-\langle++|M| \quad+\rangle$ & $=\varphi_{5}=\langle--|M|+\rangle$ & 1 & 0 \\
\hline$\langle++|M| \ldots-\rangle$ & $=\varphi_{2}=\langle--|M|++\rangle$ & 0 & 2 \\
\hline$-\langle+-|M|++\rangle$ & $=\varphi_{5}=\langle-+|M|--\rangle$ & 1 & 0 \\
\hline$\langle+-|M|+-\rangle$ & $=\varphi_{3}=\langle-+|M|-+\rangle$ & 0 & 0 \\
\hline$\langle+-|M|-+\rangle$ & $=\varphi_{4}=\langle-+|M|+-\rangle$ & 2 & 0 \\
\hline$-\langle+-|M|--\rangle$ & $=\varphi_{5}=\langle-+|M|++\rangle$ & 1 & 0 \\
\hline
\end{tabular}
zation states in terms of the amplitudes $\varphi_{1}$ An unpolarized beam or target state

Table 1

s-channel helicity amplitudes $\left(\lambda_{\mathrm{c}} \lambda_{\mathrm{d}}|M| \lambda_{\mathrm{a}} \lambda_{\mathrm{b}}\right)$ for pp elastic scattering, and their associated helicity flip quantum numbers $n=\left|\left(\lambda_{\mathrm{a}}-\lambda_{\mathrm{b}}\right)-\left(\lambda_{\mathrm{c}}-\lambda_{\mathrm{d}}\right)\right|$ and $x=\left|\lambda_{\mathrm{c}}-\lambda_{\mathrm{a}}\right|+\left|\lambda_{\mathrm{d}}-\lambda_{\mathrm{b}}\right|-n$ 
is denoted by the asterisk symbol * The total cross section for an unpolarized beam and target is

$$
\begin{aligned}
\sigma_{\mathrm{T}} & \equiv \sigma_{\mathrm{T}}(* *)=\left.\frac{-1}{2 q w} 2 \frac{1}{2 S_{\mathrm{a}}+1} 2 \frac{1}{2 S_{\mathrm{b}}+1} \sum_{\lambda_{\mathrm{a}} \lambda_{\mathrm{b}}} \operatorname{Im}\left\langle\lambda_{\mathrm{a}} \lambda_{\mathrm{b}}|M| \lambda_{\mathrm{a}} \lambda_{\mathrm{b}}\right\rangle\right|_{t=0} \\
& =-\frac{1}{8 q w} \ddot{\operatorname{Im}}[\langle++|M|++\rangle+\langle+-|M|+-\rangle+\langle-+|M|-+\rangle+\langle-\cdots|M|-\rangle]_{t=0} \\
& =-\frac{1}{4 q w} \operatorname{Im}\left(\varphi_{1}+\varphi_{3}\right)_{t=0}
\end{aligned}
$$

using table $1, q$ and $w$ are the magnitude of the $\mathrm{cm}$ three-momentum and the total energy.

Other currently measurable total cross sections with the polarized beam polarized target facility are similarly obtained using the optical theorem If only the beam or the target is transversely polarized, no new information is gained, since

$$
\sigma_{\mathrm{T}}\left({ }^{\uparrow}\right)=\sigma_{\mathrm{T}}\left({ }_{\downarrow}^{\uparrow}\right)=\sigma_{\mathrm{T}}(* *) .
$$

However, if both the beam and target are transversely polarized, we obtain from eqs (4) that

$$
\begin{aligned}
& \sigma_{\mathrm{T}}(\uparrow \uparrow)=\frac{--1}{2 q w} \operatorname{Im}\langle\uparrow \uparrow|M| \uparrow \uparrow\rangle_{t=0} \\
& =\frac{1}{4 q w} \operatorname{Im}\left(\varphi_{1}+\varphi_{3}+\varphi_{2}\right)_{t=0}=\sigma_{\mathrm{T}}(\downarrow \downarrow), \\
& \sigma_{\mathrm{T}}(\uparrow \downarrow)=\frac{1}{2 q w} \operatorname{Im}\langle\uparrow \downarrow|M| \uparrow \downarrow\rangle_{t=0}=-\frac{1}{4 q w} \operatorname{Im}\left(\varphi_{1}+\varphi_{3} \cdots \varphi_{2}\right)_{t=0}=\sigma_{\mathrm{T}}(\downarrow \uparrow),
\end{aligned}
$$

or, equivalently

$$
\begin{aligned}
& \sigma_{\mathrm{T}}(\uparrow \uparrow)+\sigma_{\mathrm{T}}(\uparrow \downarrow)=\frac{-1}{2 q w} \operatorname{Im}\left(\varphi_{1}+\varphi_{3}\right)_{t=0}=2 \sigma_{T}, \\
& \sigma_{\mathrm{T}}(\uparrow \uparrow)-\sigma_{\mathrm{T}}(\uparrow \downarrow)=\frac{-1}{2 q w}\left(\operatorname{Im} \varphi_{2}\right)_{t=0}
\end{aligned}
$$

Eq (8) provides a useful check on normalizations for the polarized beam. Eq (9) says the difference of polarized cross sections measures the imaginary part of the double flip amplitude at $t=0$, we will discuss the implication of this in sect $4(\mathrm{Eq}$ (9) was also known to Buttımore [2] )

For completeness, we give below the results corresponding to beam and target in helicity eigenstates As previously mentioned, such experiments are not currently possible, but will become so in the near future (We again remind the reader that the target in helicity eigenstates \pm corresponds to eigenvalues $S_{z}=\mp \frac{1}{2} \hbar$.) 


$$
\begin{aligned}
& \sigma_{\mathrm{T}}(* \pm)=\sigma_{\mathrm{T}}\left( \pm^{*}\right)=\sigma_{\mathrm{T}}\left( \pm \begin{array}{c}
\uparrow \\
\downarrow
\end{array}\right)=\sigma_{\mathrm{T}}\left(\begin{array}{l}
\uparrow \\
\downarrow
\end{array}\right)=\sigma_{\mathrm{T}}(* *), \\
& \sigma_{\mathrm{T}}(++)=\sigma_{\mathrm{T}}(-)=\frac{1}{4 q w}\left(\operatorname{Im} \varphi_{1}\right)_{t=0}, \\
& \sigma_{\mathrm{T}}(+-)=\sigma_{\mathrm{T}}(+)=\frac{1}{4 q w}\left(\operatorname{Im} \varphi_{3}\right)_{t=0}
\end{aligned}
$$

Eqs (11) offer the possibility of determining $\operatorname{Im} \varphi_{1}$ and $\operatorname{Im} \varphi_{3}$ separately at $t=0$ This will be a good test of the absorption assumption discussed in sect 4 , which predicts that $\varphi_{1}=\varphi_{3}[3]$

\section{Differential elastic cross sections}

The usual differential cross section for an unpolarized beam and target is

$$
\begin{aligned}
\underset{\mathrm{d} t}{\mathrm{~d} \sigma} & \equiv \underset{\mathrm{d} t}{\mathrm{~d} \sigma}(* *)=\underset{64 \pi q^{2} s}{-1} \frac{1}{2 S_{\mathrm{a}}+\overline{1}} 2 S_{\mathrm{b}}+1 \sum_{\substack{\lambda_{\mathrm{d}} \lambda_{\mathrm{b}} \\
\lambda_{\mathrm{c}} \lambda_{\mathrm{d}}}}\left|\left\langle\lambda_{\mathrm{c}} \lambda_{\mathrm{d}}|M| \lambda_{\mathrm{a}} \lambda_{\mathrm{b}}\right\rangle\right|^{2} \\
& =\underset{128 \pi q^{2} s}{.1}\left[\left|\varphi_{1}\right|^{2}+\left|\varphi_{2}\right|^{2}+\left|\varphi_{3}\right|^{2}+\left|\varphi_{4}\right|^{2}+4\left|\varphi_{5}\right|^{2}\right]
\end{aligned}
$$

For a transversely polarized target and an unpolarized beam

$$
\begin{aligned}
& \frac{\mathrm{d} \sigma}{\mathrm{d} t}\left({ }^{\uparrow}{ }_{\downarrow}^{\uparrow}\right)=\frac{1}{64 \pi q^{2}} \cdot \frac{1}{2 S_{\mathrm{a}}+1} \sum_{\lambda_{\mathrm{a}}}\left|\left\langle\lambda_{\mathrm{c}} \lambda_{\mathrm{d}}|M| \lambda_{\mathrm{a}} \uparrow\right\rangle\right|^{2} \\
& =\frac{1}{256 \pi q^{2} s} \sum_{\lambda_{\mathrm{d}}}\left|\left\langle\lambda_{\mathrm{c}} \lambda_{\mathrm{d}}|M| \lambda_{\mathrm{a}}-\lambda_{\mathrm{d}}\right\rangle \pm\left\langle\lambda_{\mathrm{c}} \lambda_{\mathrm{d}}|M| \lambda_{\mathrm{a}}+\right\rangle\right|^{2} \\
& \quad \lambda_{\mathrm{c}} \lambda_{\mathrm{d}} \\
& =\frac{1}{128 \pi q^{2} s}\left[\left|\varphi_{1} \mp l \varphi_{5}\right|^{2}+\left|\varphi_{2} \mp t \varphi_{5}\right|^{2}+\left|\varphi_{3} \mp t \varphi_{5}\right|^{2}+\left|\varphi_{4} \pm t \varphi_{5}\right|^{2}\right] \\
& =\frac{\mathrm{d} \sigma}{\mathrm{d} t}(* *) \pm \frac{1}{64 \pi q^{2} s} \operatorname{Im}\left[\left(\varphi_{1}+\varphi_{2}+\varphi_{3} \cdot \varphi_{4}\right)^{*} \varphi_{5}\right]
\end{aligned}
$$

The "standard" polanzation $P$ (left-right asymmetry on a transversely polarized target) is given by

$$
P=\frac{\mathrm{d} \sigma / \mathrm{d} t(* \uparrow, \theta)-\mathrm{d} \sigma / \mathrm{d} t(* \uparrow,-\theta)}{\mathrm{d} \sigma / \mathrm{d} t(* \uparrow, \theta)+\frac{\mathrm{d} \sigma / \mathrm{d} t(* \uparrow,-\theta)}{\theta}}
$$

Noting that $\varphi_{5}$ is the only odd amplitude under $\theta \rightarrow-\theta$, it follows that 


$$
\mathrm{d} \sigma(* \uparrow,--\theta)=\frac{\mathrm{d} \sigma}{\mathrm{d} t}(*-\downarrow, \theta)
$$

which has been calculated in eq (13) Thus, one can also define $P$ by the equation

$$
P=\frac{\mathrm{d} \sigma / \mathrm{d} t(* \uparrow) \cdot \mathrm{d} \sigma / \mathrm{d} t(* \downarrow)}{\mathrm{d} \sigma / \mathrm{d} t(* \uparrow)+\mathrm{d} \sigma / \mathrm{d} t\left({ }^{*} \downarrow\right)}
$$

A familar expression for $P$ in terms of the amplitudes $\varphi_{l}$ follows immediately by substituting eq (13) into eq (15)

$$
P=\frac{2 \operatorname{lm}\left|\left(\varphi_{1}+\varphi_{2}+\varphi_{3} \varphi_{4}\right)^{*} \varphi_{5}\right|}{\left|\varphi_{1}\right|^{2}+\left|\varphi_{2}\right|^{2}+\left|\varphi_{3}\right|^{2}+\left|\varphi_{4}\right|^{2}+4\left|\varphi_{5}\right|^{2}}
$$

The scattering of a transversely polarized beam on an unpolarized target yields the same results as above (the two processes are identical in the $\mathrm{cm}$ frame)

$$
\overline{\mathrm{d} \sigma}(\uparrow *)=\frac{\mathrm{d} \sigma}{\mathrm{d} t}(* \uparrow), \quad \frac{\mathrm{d} \sigma}{\mathrm{d} t}(\downarrow *)=\frac{\mathrm{d} \sigma}{\mathrm{d} t}\left({ }^{*} \downarrow\right)
$$

Let us now consider the interesting case in which both the beam and the target are transversely polarized Invariance under rotatıons of $180^{\circ}$ about the $Y$-axis gives

$$
\frac{\mathrm{d} \sigma}{\mathrm{d} t}(\uparrow \downarrow)=\frac{\mathrm{d} \sigma}{\mathrm{d} t}(\downarrow \uparrow)
$$

Using table 1 and eqs (4) for the two-particle initial state, we find

$$
\begin{aligned}
& \frac{d \sigma}{d t}(\uparrow \downarrow)=\frac{1}{64 \pi q^{2} s} \sum_{\lambda_{c} \lambda_{d}}\left|\left\langle\lambda_{c} \lambda_{d}|M| \uparrow \downarrow\right\rangle\right|^{2} \\
& =\left.\frac{1}{64 \pi q^{2} s}\left[\left.|K++| M|\uparrow \downarrow\rangle\right|^{2}+K+-|M| \uparrow \downarrow\right\rangle\right|^{2} \\
& \left.\left.+K_{-}+|M| \uparrow \downarrow\right\rangle\left.\right|^{2}+K^{-} \quad-|M| \uparrow \downarrow\right\rangle\left.\right|^{2} \mid \\
& =\frac{-}{256 \pi q^{2} s} \cdot\left[\mid \imath\langle++|M|++\rangle+\langle++|M|+\rangle\langle+|M|+\rangle+\imath\left\langle++\left.|M| \quad\right|^{2}\right.\right. \\
& +\quad] \\
& \left.=\left.\frac{1}{128 \pi q^{2} s}|| \varphi_{1} \varphi_{2}\right|^{2}+\left|\varphi_{3}+\varphi_{4}\right|^{2}\right]
\end{aligned}
$$

Similarly,

$$
\mathrm{d} \sigma \quad(\uparrow \uparrow)=\frac{1}{128 \pi q^{2} s}\left[\left|\varphi_{1}+\varphi_{2}-2 l \varphi_{5}\right|^{2}+\left|\varphi_{3}-\varphi_{4}-2 l \varphi_{5}\right|^{2}\right]
$$




$$
\frac{\mathrm{d} \sigma}{\mathrm{d} t}(\downarrow \downarrow)=-\frac{1}{128 \pi q^{2} s}-\left[\left|\varphi_{1}+\varphi_{2}+2 \imath \varphi_{5}\right|^{2}+\left|\varphi_{3}-\varphi_{4}+2 \imath \varphi_{5}\right|^{2}\right]
$$

Equivalently,

$$
\begin{aligned}
& \frac{\mathrm{d} \sigma}{\mathrm{d} t}(\uparrow \uparrow)+\frac{\mathrm{d} \sigma}{\mathrm{d} t}(\uparrow \downarrow)=2(1+P) \frac{\mathrm{d} \sigma}{\mathrm{d} t}, \\
& \frac{\mathrm{d} \sigma}{\mathrm{d} t}(\uparrow \uparrow)-\frac{\mathrm{d} \sigma}{\mathrm{d} t}(\downarrow \downarrow)=4 P \frac{\mathrm{d} \sigma}{\mathrm{d} t},
\end{aligned}
$$

which can serve as a useful consistency check or give good measurements of $P$, and

$$
\begin{gathered}
\Delta\left(\begin{array}{l}
\mathrm{d} \sigma \\
\overline{\mathrm{d} t}
\end{array}\right) \equiv \frac{\mathrm{d} \sigma}{\mathrm{d} t}(\uparrow \uparrow)+\frac{\mathrm{d} \sigma}{\mathrm{d} t}(\downarrow \downarrow)-\frac{\mathrm{d} \sigma}{\mathrm{d} t}(\uparrow \downarrow)-\frac{\mathrm{d} \sigma}{\mathrm{d} t} \\
=\frac{1}{16 \pi q^{2} s}\left[2\left|\varphi_{5}\right|^{2}+\operatorname{Re}\left(\varphi_{1} \varphi_{2}^{*} \cdots \varphi_{3} \varphi_{4}^{*}\right)\right]
\end{gathered}
$$

The quantity $2\left|\varphi_{5}\right|^{2}+\operatorname{Re}\left(\varphi_{1} \varphi_{2}^{*}-\varphi_{3} \varphi_{4}^{*}\right)$ is the new information one obtains from polarized beam and target experiments for $\mathrm{d} \sigma / \mathrm{d} t$ In particular, this quantity reduces to $\operatorname{Re} \varphi_{1} \varphi_{2}^{*}$ at $t=0$

For completeness, we discuss results for helicity eigenstates also It is amusing to note that measurements with either the beam or the target in a helicity eigenstate do not teach us anything new when summing over final state spins, since

$$
\begin{aligned}
& \frac{\mathrm{d} \sigma}{\mathrm{d} t}\left( \pm^{*}\right)=\frac{\mathrm{d} \sigma}{\mathrm{d} t}(* \pm)=\frac{\mathrm{d} \sigma}{\mathrm{d} t}(* *), \\
& \frac{\mathrm{d} \sigma}{\mathrm{d} t}\left(\begin{array}{l}
\uparrow \\
+
\end{array}\right)=\frac{\mathrm{d} \sigma}{\mathrm{d} t}\left({ }_{\downarrow}^{\uparrow}-\right)=\frac{\mathrm{d} \sigma}{\mathrm{d} t}\left({ }_{\downarrow}^{\uparrow}\right)
\end{aligned}
$$

However, measurements with beam and target both in helicity eigenstates do yield new information

$$
\begin{aligned}
& \frac{\mathrm{d} \sigma}{\mathrm{d} t}(++)=\frac{\mathrm{d} \sigma}{\mathrm{d} t}(--)=\frac{1}{64 \pi q^{2} s}\left[\left|\varphi_{1}\right|^{2}+\left|\varphi_{2}\right|^{2}+2\left|\varphi_{5}\right|^{2}\right], \\
& \frac{\mathrm{d} \sigma}{\mathrm{d} t}(+-)=\frac{\mathrm{d} \sigma}{\mathrm{d} t}(-+)=\frac{1}{64 \pi q^{2} s}\left[\left|\varphi_{3}\right|^{2}+\left|\varphi_{4}\right|^{2}+2\left|\varphi_{5}\right|^{2}\right],
\end{aligned}
$$

or equivalently,

$$
\frac{\mathrm{d} \sigma}{\mathrm{d} t}(++)+\frac{\mathrm{d} \sigma}{\mathrm{d} t}(+-)=2 \frac{\mathrm{d} \sigma}{\mathrm{d} t}(* *)
$$


Table 2

A summary of new information which can be obtaned from pp total and differental cross sections using polarized beams and targets

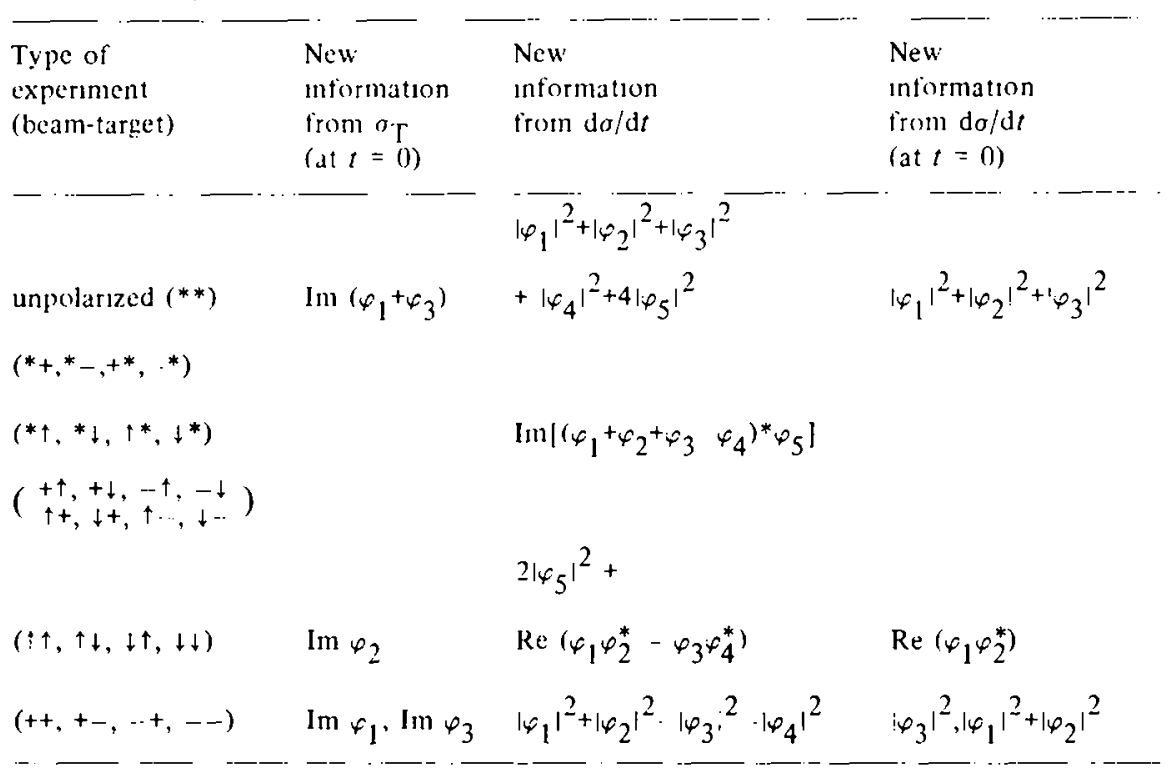

More information can be had in the forward direction because of the optical theorem and the fact that only $\varphi_{1}, \varphi_{2}, \varphi_{3}$ can be non-rero at $t=0$ (currently feasible measurements at $t=0$ of $\mathrm{d} \sigma / \mathrm{d} t, \sigma_{\mathrm{T}}, \sigma_{\mathrm{T}}(\uparrow \uparrow)-\sigma_{\mathrm{T}}(\uparrow \downarrow), 2 \mathrm{~d} \sigma(\uparrow \downarrow) / \mathrm{d} t-\mathrm{d} \sigma(\uparrow \uparrow) / \mathrm{d} t-\mathrm{d} \sigma(\downarrow \downarrow) / \mathrm{d} t$, and the Coulomb interference are enough to determine $\varphi_{1}$ and $\varphi_{2}$ fully if the basic $s$-channel absorption assumption that $\varphi_{1}=\varphi_{3}$ is made Measurements with beam and target longitudinally polarized (helicity cugenstates) then test the assumption $\varphi_{1}=\varphi_{3}$

$$
\frac{\mathrm{d} \sigma}{\mathrm{d} t}(++)-\frac{\mathrm{d} \sigma}{\mathrm{d} t}(+-\cdot)=-\frac{1}{64 \pi q^{2}} ; \quad\left[\left|\varphi_{1}\right|^{2}+\left|\varphi_{2}\right|^{2}-\left|\varphi_{3}\right|^{2}-\left|\varphi_{4}\right|^{2}\right]
$$

Thus differential cross-section measurements with helicity eigenstates give the new combination $\left|\varphi_{1}\right|^{2}+\left|\varphi_{2}\right|^{2}-\left|\varphi_{3}\right|^{2}-\left|\varphi_{4}\right|^{2}$, which reduces to $\left|\varphi_{1}\right|^{2}+\left|\varphi_{2}\right|^{2}-$ $\left|\varphi_{3}\right|^{2}$ at $t=0$ A summary of new information from polarized beam-target experments is given in table 2

\section{The spectal point $t=0$}

The point $t=0$ is especially interesting for two reasons (1) More information is avalable here from total cross section measurements and the optical theorem.

(11) There are less amplitudes, since $\varphi_{4}(t=0)=\varphi_{5}(t=0)=0$

In particular, if we make the assumption that $\varphi_{1}=\varphi_{3}$, whose possible validity is discussed in sect 4 , there are only two (complex) amplitudes to determine, say $\varphi_{1}$ and $\varphi_{2}$ The avalable data from the current set of experiments will be 
$\begin{array}{ll}\text { (a) } \sigma_{\mathrm{T}} & =\frac{1}{2 q w} \operatorname{Im} \varphi_{1}, \\ \text { (b) } \Delta \sigma_{\mathrm{T}} & =\sigma_{\mathrm{T}}(\uparrow \uparrow)-\sigma_{\mathrm{T}}(\uparrow \downarrow)=\frac{1}{2 q w} \operatorname{Im} \varphi_{2}, \\ \text { (c) } \mathrm{d} \sigma / \mathrm{d} t & =\left(2\left|\varphi_{1}\right|^{2}+\left|\varphi_{2}\right|^{2}\right) / 128 \pi q^{2} s, \\ \text { (d) } \Delta(\mathrm{d} \sigma / \mathrm{d} t) & =\operatorname{Re} \varphi_{1} \varphi_{2}^{*} / 16 \pi q^{2} s \text { from eq (23). } \\ \text { (c) } \operatorname{Re} \varphi_{1} / \operatorname{Im} \varphi_{1} \text { from Coulomb interference measurements }\end{array}$

The quantities (a) - (e) fully determine $\varphi_{1}$ and $\varphi_{2}$ at $t=0$, including the $a b$ solute phase The Coulomb interference measurement of the real to imaginary ratio of the forward amplitude really measures something more complicated involving other amplitudes, but above a few $\mathrm{GeV} / \mathrm{c}$ it should be quite safe to assume it is $\operatorname{Re} \varphi_{1}$ that is interfering, given a set of amplitudes to interfere with the known Coulomb helicity amplitudes one can easily check

In principle one can do even a little better, because (c) is redundant [get Im $\varphi_{1}$ from (a), Im $\varphi_{2}$ from (b), $\operatorname{Re} \varphi_{1}$ from (e), and $\operatorname{Re} \varphi_{2}$ from (d)] Then the validity of (c) is already a check on the correctness of the assumption $\varphi_{1}=\varphi_{3}$ The quadratic nature of (c) does not allow a determmation just using (a) (c) and $(e)$, although it does restrict $\operatorname{Re} \varphi_{2}$ considerably

\section{3. pp $\rightarrow \Delta^{++} n$}

[et us now consider the inelastic reaction $p p \rightarrow \Delta^{++} n[5,6]$ After applying parity constraints, there are sixteen independent amplitudes $\psi_{l}$, which are given in table 3 The decay $\Delta^{++} \rightarrow \pi^{+} p$ is experimentally measured, and it is possible to extract the following production density matrix elements [5]

$$
\rho_{\frac{3}{2} \frac{3}{2}}, \quad \rho_{\frac{1}{2} \frac{1}{2}}, \quad \operatorname{Re} \rho_{\frac{3}{2} \frac{1}{2}}, \quad \operatorname{Re} \rho_{\frac{3}{2}} \frac{1}{2}
$$

For various initial transverse polarization states, we express these observed density matrix element in terms of the amplitudes $\psi_{l}$ of table 3

The (unnormalized) production density matrix elements for an unpolarized beam - unpolanzed target experment are defined by

$$
\rho_{\mu \nu}(* *)=\frac{1}{4} \sum_{\lambda_{a} \lambda_{b} \lambda_{c}}\left\langle\lambda_{c} \mu|M| \lambda_{a} \lambda_{b}\right\rangle\left\langle\lambda_{c} \nu|M| \lambda_{a} \lambda_{b}\right\rangle *
$$

Note that the density matrix is manifestly Hermitian. The experimental observables yield

$$
\rho_{\frac{3}{2} \frac{3}{2}}(* *)={ }_{4}^{1}\left\{\left|\psi_{1}\right|^{2}+\left|\psi_{2}\right|^{2}+\left|\psi_{7}\right|^{2}+\left|\psi_{8}\right|^{2}+\left|\psi_{9}\right|^{2}+\left|\psi_{10}\right|^{2}+\left|\psi_{15}\right|^{2}+\left|\psi_{16}\right|^{2}\right\},
$$


Table 3

s-channel helicity amplitudes for the process $p p \rightarrow \Delta^{++} n$

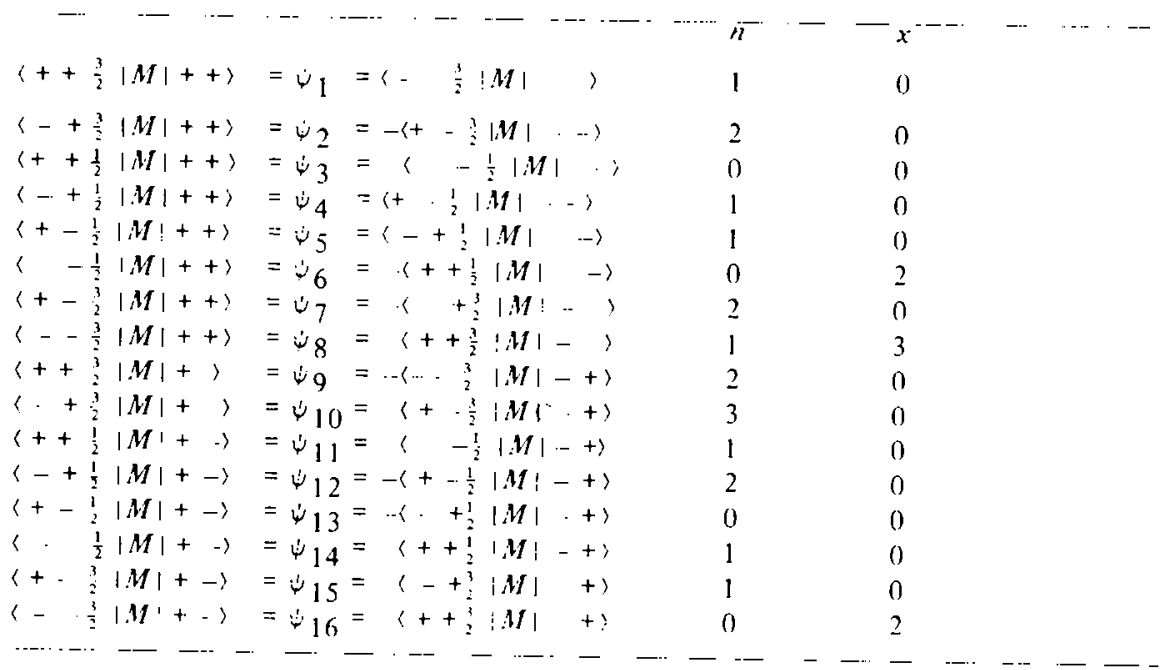
The notation used is $\left(\lambda_{\mathrm{n}} \lambda_{\Delta} ! M \mid \lambda_{\mathrm{a}} \lambda_{\mathrm{b}}\right)$, where $\lambda_{\mathrm{a}}=$ helicity of beam proton, $\lambda_{\mathrm{b}}=$ helicity of tar-
get proton

$$
\begin{aligned}
& \rho_{\frac{1}{2} !},(* *)={ }_{4}^{1}\left\{\left|\psi_{3}\right|^{2}+\left|\psi_{4}\right|^{2}+\left|\psi_{5}\right|^{2}+\left|\psi_{6}\right|^{2}\right. \\
& \left.+\left|\psi_{11}\right|^{2}+\left|\psi_{12}\right|^{2}+\left|\psi_{13}\right|^{2}+\left|\psi_{14}\right|^{2}\right\} \\
& \operatorname{Re} \rho_{\frac{3}{\Sigma}, \frac{1}{2}}(* *)=\operatorname{Re} \stackrel{1}{i}\left[\left\{\psi_{1} \psi_{3}^{*}+\psi_{2} \psi_{4}^{*}+\psi_{9} \psi_{11}^{*}+\psi_{10} \psi_{12}^{*}\right\}\right. \\
& \left.-\left\{\psi_{5}^{*} \psi_{7}+\psi_{6}^{*} \psi_{8}+\psi_{13}^{*} \psi_{15}+\psi_{14}^{*} \psi_{16}\right\}\right] \text {, } \\
& \operatorname{Re} \rho_{3-1}(* *)=\operatorname{Re}_{4}^{1}\left\{\left\{\psi_{1} \psi_{5}^{*}+\psi_{2} \psi_{6}^{*}+\psi_{9} \psi_{13}^{*}+\psi_{10} \psi_{14}^{*}\right\}\right. \\
& \left.+\left\{\psi_{3}^{*} \psi_{7}+\psi_{4}^{*} \psi_{8}+\psi_{11}^{*} \psi_{15}+\psi_{12}^{*} \psi_{16}\right\}\right]
\end{aligned}
$$

A transversely polarized beam-unpolarized target $\left(\uparrow_{\downarrow}^{\uparrow}\right)$ experiment has density matrix elements

$$
\rho_{\mu \nu}\left(\downarrow^{*}\right)=\frac{1}{2} \sum_{\lambda_{b} \lambda_{c}}\left\langle\lambda_{c} \mu|M| \uparrow_{\downarrow}^{\uparrow} \lambda_{b}\right\rangle\left\langle\lambda_{c} \nu|M|_{\downarrow}^{\uparrow} \lambda_{b}\right\rangle^{*},
$$

and the observed quantities are

$$
\begin{aligned}
& \left.\rho_{\frac{3}{2} \frac{3}{2}}\left(\begin{array}{l}
\uparrow * \\
\downarrow
\end{array}\right)=\left.\frac{1}{4}|| \psi_{1} \mp l \psi_{16}\right|^{2}+\left|\psi_{2} \pm l \psi_{15}\right|^{2}+\left|\psi_{8} \mp l \psi_{9}\right|^{2}+\left|\psi_{7} \pm l \psi_{10}\right|^{2}\right], \\
& \rho_{\frac{1}{2} \frac{1}{2}}\left(\begin{array}{l}
\uparrow \\
\downarrow
\end{array}\right)=\frac{1}{4}\left[\left|\psi_{3} \pm l \psi_{14}\right|^{2}+\left|\psi_{4} \mp l \psi_{13}\right|^{2}+\left|\psi_{5} \mp l \psi_{12}\right|+\left|\psi_{6} \pm l \psi_{11}\right|^{2}\right],
\end{aligned}
$$




$$
\begin{aligned}
& \operatorname{Re} \rho_{3,},\left(\begin{array}{l}
\uparrow \\
\downarrow
\end{array}\right)=\operatorname{Re} \frac{1}{4}\left[\left(\psi_{1} \mp l \psi_{16}\right)\left(\psi_{3}^{*} \mp \imath \psi_{14}^{*}\right)+\left(\psi_{9} \pm \imath \psi_{8}\right)\left(\psi_{11}^{*} \pm l \psi_{6}^{*}\right)\right. \\
& \left.+\left(\psi_{2} \pm \imath \psi_{15}\right)\left(\psi_{4}^{*} \pm \imath \psi_{i 3}^{*}\right)+\left(\psi_{10} \mp l \psi_{7}\right)\left(\psi_{12}^{*} \mp l \psi_{5}^{*}\right)\right],
\end{aligned}
$$

$\operatorname{Re} \rho_{\frac{3}{2} \cdot \frac{1}{2}}\left(\begin{array}{l}\uparrow \\ \downarrow\end{array}\right)=\operatorname{Re} \frac{1}{4}\left[\left(\psi_{1} \mp l \psi_{16}\right)\left(\psi_{5}^{*} \pm l \psi_{12}^{*}\right)+\left(\psi_{9} \pm l \psi_{8}\right)\left(\psi_{13}^{*} \mp l \psi_{4}^{*}\right)\right.$

$$
\left.+\left(\psi_{2} \pm \imath \psi_{15}\right)\left(\psi_{6}^{*} \mp \imath \psi_{11}^{*}\right)+\left(\psi_{10} \mp \imath \psi_{7}\right)\left(\psi_{14}^{*} \pm \imath \psi_{3}^{*}\right)\right) \text {. }
$$

Similarly, an unpolarized beam -- transversely polarized target $\left(*_{\downarrow}^{\uparrow}\right)$ experiment has density matrix elements

$$
\rho_{\mu \nu}\left(*_{\downarrow}^{\uparrow}\right)=\frac{1}{2} \sum_{\lambda_{\mathrm{a}} \lambda_{\mathrm{c}}}\left\langle\lambda_{\mathrm{c}} \mu|M| \lambda_{\mathrm{a} \downarrow}^{\uparrow}\right\rangle\left\langle\lambda_{\mathrm{c}} \nu|M| \lambda_{\mathrm{a}} \uparrow_{\downarrow}^{\dagger},\right.
$$

and the observed quantities are

$$
\begin{aligned}
& \rho_{\frac{3}{2} \frac{3}{2}}\left(*^{\uparrow}\right)=\frac{1}{4}\left[\left|\psi_{1} \mp l \psi_{9}\right|^{2}+\left|-\psi_{16} \mp \imath \psi_{8}\right|^{2}+\left|\psi_{2} \mp \imath \psi_{10}\right|^{2}+\left|\psi_{15} \pm \imath \psi_{7}\right|^{2}\right] \text {, } \\
& \rho_{\frac{1}{2} \frac{1}{2}}\left(* *_{\downarrow}^{\uparrow}\right)=\frac{1}{4}\left[\left|\psi_{3} \mp l \psi_{11}\right|^{2}+\left|\psi_{14} \pm \imath \psi_{6}\right|^{2}+\left|\psi_{4} \mp l \psi_{12}\right|^{2}+\left|\cdots \psi_{13} \mp l \psi_{5}\right|^{2}\right] \text {, } \\
& \operatorname{Re} \rho_{\frac{3}{2} \frac{1}{2}}\left(*_{\downarrow}^{\uparrow}\right)=\operatorname{Re} \frac{1}{4}\left[\left(\psi_{1} \mp l \psi_{9}\right)\left(\psi_{3}^{*} \pm l \psi_{11}^{*}\right)+\left(-\psi_{16} \mp l \psi_{8}\right)\left(\psi_{14}^{*} \mp \imath \psi_{6}^{*}\right)\right. \\
& \left.+\left(\psi_{2} \mp \imath \psi_{10}\right)\left(\psi_{4}^{*} \pm \imath \psi_{12}^{*}\right)+\left(\psi_{15} \pm \imath \psi_{7}\right)\left(--\psi_{13}^{*} \pm \imath \psi_{5}^{*}\right)\right] \text {, } \\
& \operatorname{Re} \rho_{\frac{3}{2}}-\frac{1}{2}\left(*_{\downarrow}^{\uparrow}\right)=\operatorname{Re} \frac{1}{4}\left[\left(\psi_{1} \mp l \psi_{9}\right)\left(\psi_{5}^{*} \pm l \psi_{13}^{*}\right)+\left(\cdots \psi_{16} \mp l \psi_{8}\right)\left(-\psi_{12}^{*} \pm i \psi_{4}^{*}\right)\right. \\
& \left.+\left(\psi_{2} \mp l \psi_{10}\right)\left(\psi_{6}^{*} \pm \imath \psi_{14}^{*}\right)+\left(\psi_{15} \pm l \psi_{7}\right)\left(\psi_{11}^{*} \mp l \psi_{3}^{*}\right)\right]
\end{aligned}
$$

Finally, when both the beam and target are transversely polarized, the production density matrix is

$$
\rho_{\mu \nu}\left(\begin{array}{c}
\uparrow \uparrow \\
\downarrow \downarrow
\end{array}\right)=\sum_{\lambda_{c}}\left\langle\lambda_{c} \mu|M|_{\downarrow \downarrow}^{\uparrow \uparrow}\right\rangle\left\langle\lambda_{c} \nu|M|_{\downarrow \downarrow}^{\uparrow \uparrow}\right\rangle^{*},
$$

and the observed quantities are

$$
\begin{aligned}
& \rho_{\frac{3}{2} \frac{3}{2}}\left(\uparrow_{\downarrow}^{\uparrow}\right)=\frac{1}{4}\left[\left|\psi_{1} \pm \psi_{8} \mp l \psi_{9}-\imath \psi_{16}\right|^{2}+\left|\psi_{2} \mp \psi_{7} \mp l \psi_{10}+l \psi_{15}\right|^{2}\right],
\end{aligned}
$$

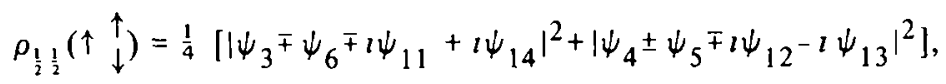

$$
\begin{aligned}
& \operatorname{Re} \rho_{\frac{3}{2} \frac{1}{2}}\left(\uparrow \uparrow_{\downarrow}^{\uparrow}\right)=\operatorname{Re}_{4}^{1}\left[\left(\psi_{1} \pm \psi_{8} \mp l \psi_{9}^{-l l} \psi_{16}\right)\left(\psi_{3}^{*} \mp \psi_{6}^{*} \pm l \psi_{11}^{*}-l \psi_{14}^{*}\right)\right. \\
& \left.+\left(\psi_{2} \mp \psi_{7} \mp l \psi_{10}+l \psi_{15}\right)\left(\psi_{4}^{*} \pm \psi_{5}^{*} \pm \imath \psi_{12}^{*}+\imath \psi_{13}^{*}\right)\right] \text {, }
\end{aligned}
$$




$$
\begin{aligned}
& \operatorname{Re} \rho_{2}-\frac{1}{2}\left(\uparrow \begin{array}{c}
\uparrow \\
\downarrow
\end{array}\right)=\operatorname{Re}{ }_{i}^{\prime}\left[\left(\psi_{1} \pm \psi_{8} \mp l \psi_{9}-l \psi_{16}\right)\left(\psi_{5}^{*} \pm \psi_{4}^{*} \pm l \psi_{13}^{*}+l \psi_{12}^{*}\right)\right. \\
& \left.+\left(\psi_{2} \mp \psi_{7} \mp l \psi_{10}+l \psi_{15}\right)\left(\psi_{6}^{*} \mp \psi_{3}^{*} \pm l \psi_{14}^{*}-l \psi_{11}^{*}\right)\right],
\end{aligned}
$$

and similarly

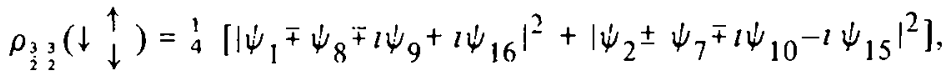

$$
\begin{aligned}
& \rho_{\frac{1}{2} \frac{1}{2}}\left(\downarrow \begin{array}{l}
\uparrow \\
\downarrow
\end{array}\right)=\frac{1}{4}\left[\left|\psi_{3} \pm \psi_{6} \mp l \psi_{11}-l \psi_{14}\right|^{2}+\left|\psi_{4} \mp \psi_{5} \mp l \psi_{12}+l \psi_{13}\right|^{2}\right], \\
& \operatorname{Re} \rho_{\frac{3}{2} \frac{1}{2}}\left(\downarrow \downarrow_{\downarrow}^{\uparrow}\right)=\operatorname{Re}{ }_{4}^{1}\left[\left(\psi_{1} \mp \psi_{8} \mp l \psi_{9}+\imath \psi_{16}\right)\left(\psi_{3}^{*} \pm \psi_{6}^{*} \pm \imath \psi_{11}^{*}+\imath \psi_{14}^{*}\right)\right. \\
& \left.+\left(\psi_{2} \pm \psi_{7} \mp i \psi_{10} \cdot l \psi_{15}\right)\left(\psi_{4}^{*} \mp \psi_{5}^{*} \pm l \psi_{12}^{*} \cdot l \psi_{13}^{*}\right)\right], \\
& \operatorname{Re} \rho_{\frac{3}{2}-\frac{1}{2}}\left(\downarrow \begin{array}{l}
\uparrow \\
\downarrow
\end{array}\right)=\operatorname{Re}{ }_{4}^{1}\left[\left(\psi_{1} \mp \psi_{8} \mp \imath \psi_{9}+\imath \psi_{16}\right)\left(\psi_{5}^{*} \mp \psi_{4}^{*} \pm l \psi_{13}^{*}-\imath \psi_{12}^{*}\right)\right. \\
& \left.+\left(\psi_{2} \pm \psi_{7} \mp l \psi_{10}-l \psi_{15}\right)\left(\psi_{6}^{*} \pm \psi_{3}^{*} \pm \imath \psi_{14}^{*}+l \psi_{11}^{*}\right)\right]
\end{aligned}
$$

Since we have 16 complex amplitudes $\psi_{l}$, even the wealth of information described above (a lot of it is redundant) is insufficient to disentangle them Instead, it is much more fruitful to reduce the number of amplitudes in a useful way, for example by making the absorption assumption given in sect. $4 \mathrm{~A}$ point of special interest is $t=0$ Here, angular momentum conservation requires that all $\psi_{\text {, except } l}=3,6,13,16$ must vanish The information from experiments at $t=0$ is given in table 4 The absorption assumption gives $\psi_{3}=\psi_{13}$, since they both have the same $n$ and $x$ values. Measurements will yield the following quantities at $t=0\left|\psi_{16}\right|^{2}, 2\left|\psi_{3}\right|^{2}+\left|\psi_{6}\right|^{2}, \operatorname{Im}\left(\psi_{6}^{*} \psi_{16}\right), \operatorname{Im}\left(\psi_{3}^{*} \psi_{16}\right)$,

Table 4

A summary of new information obtainable at $t=0$ by measuring the production density matrix elements in the reaction $\mathrm{pp} \rightarrow \Delta^{++} \mathrm{n}$

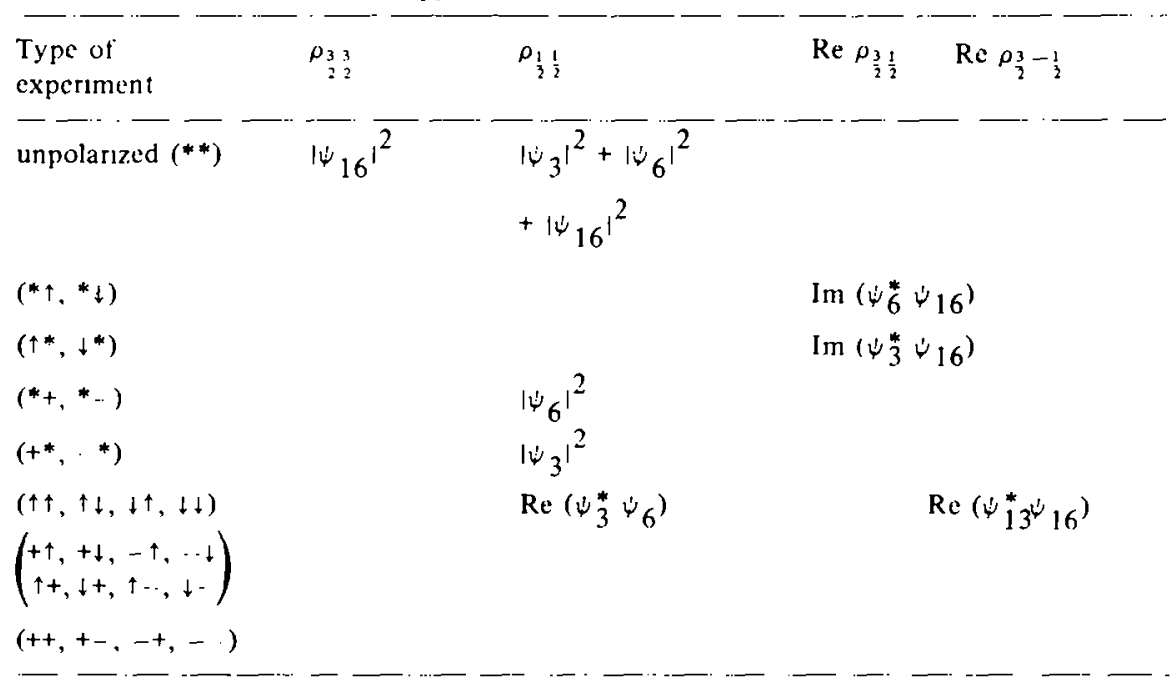

The only amplitudes which can be non-zero at $t=0$ are $3 . \psi_{6} . \psi_{13}, \psi_{16}$ 
$\operatorname{Re}\left(\psi_{3}^{*} \psi_{6}\right), \operatorname{Re}\left(\psi_{3}^{*} \psi_{16}\right)$ It is easy to check that this information is sufficient to determine $\psi_{3}, \psi_{6}, \psi_{16}$ at $t=0$ up to an overall phase

\section{4. s-channel absorption assumption and numerical predictions}

In this section we first want to draw attention to the general application of a point we have made in context above It has often been noted that whenever possible, individual scattering amplitudes should be determıned from data In general it is not possible, and people have made a number of assumptions of an ad hoc nature to relate data to the behavior of amplitudes

Of the various assumptions, one [3] has a considerably greater chance of being correct than most, at the mummum, it is not yet known to be incorrect in any reaction (which is not the case for most assumptions)' It is also quite general, apphcable in many places, and eventually subject to a large number of tests. To state $1 t$ one defines net helicity flip quantum numbers $n=\left|\left(\lambda_{\mathrm{a}}-\lambda_{\mathrm{b}}\right) \cdots\left(\lambda_{\mathrm{c}} \lambda_{\mathrm{d}}\right)\right|$ and $x=\left|\lambda_{c}-\lambda_{\mathrm{a}}\right|+\left|\lambda_{\mathrm{d}}-\lambda_{\mathrm{b}}\right| n$ Then the basic prediction [3] of s-channel absorption is that amplitudes which would be proportional (up to a constant factor) If only definute party exchanges were present, are still proportional if they have the same $n$ and $x$. even when absorptive rescattermng effects are included

For NN $\rightarrow \mathrm{NN}$, for example, with a pomeron contribution, vector and tensor exchange, and $\pi$ exchange, $\varphi_{1}$ and $\varphi_{3}$ only get contributions from natural parity exchanges (pomeron, V, T) They have the same $n=0, x=0$, so they are assumed equal On the other hand, if there were only $\pi$ exchange the particle contribution would give $\varphi_{2}=\varphi_{4}$, but these have different $n, x$ values so one should leave $\varphi_{2}$ and $\varphi_{4}$ as independent amplitudes even in that simple case Altogether, for $\mathrm{NN} \rightarrow \mathrm{NN}$ our assumption leaves 4 independent amplitudes, with $(n, x)$ values of $(0,0),(1,0),(2,0),(0,2)$

To have $\varphi_{1} \neq \varphi_{3}$ for the NN elastic case one would need an important contribution (comparable in size to $\mathrm{V}$ or $\mathrm{T}$ ) which har unnatural parity and which did not flip s-channel helicities (in terms of particle names, $A_{1}$-like) Then it would interfere constructively with $\mathrm{V}+\mathrm{T}$ in $\varphi_{1}$ and destructively in $\varphi_{3}$, giving a difference As noted above, this can be tested by experments with longitudinal polarization

For $p p \rightarrow \Delta^{++} n$ we assume the important exchanges are $\pi, \rho, \mathrm{A}_{2}$ Then to reduce the 16 amplitudes, we need cases where opposite naturalities do not contribute and where $n, x$ are the same We get

$$
\psi_{3}=\psi_{13}, \psi_{7}=\psi_{9}, \psi_{1}=\psi_{15}, \psi_{5}=\psi_{11} \text {, }
$$

leaving still 12 amplitudes At $t=0$, however, 4 non-zero amplitudes are allowed $\left(\psi_{3}, \psi_{13}, \psi_{6}, \psi_{16}\right)$ and our assumption reduces these by one since $\psi_{3}=\psi_{13}$ As mentioned previously, current measurements of the $\Delta$ density matrix with 
transversely polarized beam and target give 6 independent numbers (see table 4), allowing the three amplitudes to be fully determined Measurements with longtudinal polarization states allow one to test whether $\psi_{3}=\psi_{13}$

\section{Remarks about $p p \rightarrow \Delta^{++} n$}

A few observations can be made here independent of detalled assumptions

(1) The quantity

$$
\begin{gathered}
\rho_{\frac{1}{2} \frac{1}{2}}(\uparrow *) \quad \rho_{1 !}\left(\downarrow^{*}\right) \\
\rho_{\frac{1}{2} \frac{1}{2}(\uparrow *)+\rho_{1}\left(\downarrow^{*}\right)}\left(\downarrow^{*}\right)
\end{gathered}=P_{11}
$$

has precisely the spm structure of the polarization in a $\frac{1}{2}++^{+} \rightarrow \frac{1}{2}++\frac{1}{2}+$ reaction Since the exchanges here are the same as in $n p \rightarrow p n$, comparison of $P$ $\left(n p \rightarrow\right.$ pn) with $P_{11}$ will tell us about any differences in behavior in couplings to $N N$ and to $N \Delta$ The expected differences do not change the polarization much. so $P_{11}$ should indeed look like $P$ (np $\rightarrow$ pn)

(11) The quantity $\rho_{\frac{3}{2}}(\uparrow *)-\rho_{3}\left(\downarrow^{*}\right)$ has the spin structure of a quantity like Re $\rho_{10}$ in $\pi \mathrm{N} \rightarrow \rho \mathrm{N}$ or $\mathrm{KN} \rightarrow \mathrm{K}^{*} \mathrm{~N}$, being dominated by an $n=1$ ampltude interfering with the sum of $n, x=2,0$ and 0,2 . By comparison, we can learn about the coupling patterns

(111) $\rho_{\frac{1}{2}, \frac{1}{3}}(* *) \mathrm{d} \sigma / \mathrm{d} t$ and $\rho_{3}(* *) \mathrm{d} \sigma / \mathrm{d} t$ have the form of $\mathrm{d} \sigma(\mathrm{np} \rightarrow \mathrm{pn}) / \mathrm{d} t$ and contain the sharp small $t$ peak characteristic of $n p \rightarrow$ pn Whether it shows in the data depends on the size of the other odd $n \pi$ exchange amplitudes in these quantities, $\left|\psi_{4}\right|^{2}+\left|\psi_{14}\right|^{2}$ in $\rho_{\frac{1}{2}}$ (these may be large) and $\left|\psi_{8}\right|^{2}+\left|\psi_{10}\right|^{2}$ in $\rho_{3} ;$ (these should be small since $n+x \geqslant 3$ )

(iv) $\rho_{3}+\frac{1}{2}(\uparrow *)-\rho_{\frac{3}{2}+\frac{1}{2}}\left(\downarrow^{*}\right)$ depends on amplitudes with $n, x=1,3$ and 3,0 which have not been studied in other reactions perhaps some hint of their behavior can be obtained here

(v) Before making any detaled calculations one could proceed in steps The next assumption to make is that the $\rho . \Lambda_{2}$ couplings are of the Stodolsky-Sakurat type [7] Rather than do precisely that we make the s-channel version. in two steps

Coupling assumption $1 \rho$ and $A_{2}$ coupling to $\mathrm{N} \Delta$ always flip $s$-channel helicities one unit (CA1) This implies

$$
\dot{\psi}_{3}=\psi_{13}=\psi_{7}=\psi_{9}=0, \quad \psi_{14}=\psi_{4} \text { and still } \psi_{1}=\psi_{15}, \psi_{5}=\psi_{11} \text {, }
$$

so there are now 9 independent amplitudes At $t=0$ only $\psi_{6}$ and $\psi_{16}$ are now non-zero, so the measurements shown in table 4 will determine them upto an overall phase, and also check the validity of CAl for $\psi_{3}$ and $\psi_{13}{ }^{\prime}$

Even better, the amplitudes $\psi_{16}$ and $\psi_{6}$ are just those needed to test the other part of the s-channel version of Stodolsky-Sakural 
Coupling assumption $2 \rho$ and $A_{2}$ couple to $N \Delta, \sqrt{3}$ times more strongly when the helicities are $\frac{1}{2} \rightarrow \frac{3}{2}$ than $\frac{\Gamma}{2} \rightarrow-\frac{1}{2}$ Thus, we expect $\psi_{16}=\sqrt{3} \psi_{6}$ (i.e $\sqrt{3}$ in magnitude and relatively real)

Away from $t=0$ detaled calculations are required to make predictions, though with the s-channel absorption assumption and CAl, 2 the number of amplitudes is down to seven (One more simplification is possible, as the $\pi$ also satisfies CA2 to a good approximation, but no useful results emerge without calculation.)

One can use a Stodolsky-Sakurat $\rho-\gamma$ analogy in several forms, they originally used a Lagrangian, while we have used it in an s-channel form, and others have used a $t$-channel form It is not possible yet to distınguish among these experimentally as they differ in contributions proportional to $t /\left(m_{\Delta}+m_{N}\right)$ or $\left(m_{\Delta}-m_{N}\right) / m_{\Delta}$ and these are too small to show up in the data If any choice is favored by the data it may be the $s$-channel one, as the other choices give an $s$-channel nonflip ( $n=0, x=0$ ) amplitude which has (after effects such as absorption are included) a sharp forward peak and has not yet been observed experımentally

\section{$42 \operatorname{Im} \varphi_{2}(0)$}

Since the measurement of $\operatorname{Im} \varphi_{2}(0)$ is one of the main results of the polarized beam experiments, we give a general but rather long and detalled set of arguments to see what value and sign it should have $A$ disagreement between our result and the data would imply that some of the following are incorrect As each step in the argument is on rather firm ground, a disagreement (as seems to be suggested) would perhaps tell us something significant Although we have taken considerable care and effort to have consistent conventions and to use the conventions of the experimenters, so many signs are involved that additıonal checking is presumably required We choose $P_{\text {lab }} \sim 6 \mathrm{GeV} / c$ where necessary

(1) We assume that the contributions are the main elastic amplitude (the pomeron), the $\pi$, and vector and tensor mesons $\rho, \omega, \mathrm{f}, \mathrm{A}_{2}$ This is presumably the weakest link in the chain since we do not have a controlled test, but there is no other place in two-body reactions at similar energies where something else is needed Thus, our result should be interpreted to give the situation for these contributions

(11) We choose the pomeron phase to be negative imaginary, $\sigma_{\mathrm{T}} \approx-\operatorname{Im} \varphi_{1} / s$ This is just a convention and does not affect the answer

(i1) Since $\sigma_{T}(p p)$ falls with energy, the reggeon contributions must also have a negative imaginary part The dominant reggeons are $\omega+f$ (known from other two-body phenomenology - e.g $\sigma_{\mathrm{T}}(\mathrm{pp}) \approx \sigma_{\mathrm{T}}(\mathrm{np})$. so isoscalar reggeons dominate)

(iv) Since pp is an exotic channel, the reggeons are almost completely real and provide most of the real part of $\varphi_{1}$ Since $\operatorname{Re} \varphi_{1} / \operatorname{Im} \varphi_{1}$ is known to be negative the reggeons $\omega+f$ have a positive real part Thus they lie in the 4 th quadrant, somewhere between the real axis and $-45^{\circ}$, at $t=0$ 
(v) By SU(3) the isovector reggeons $\rho+A_{2}$ have essentrally the same phase at $t=0$ as $\omega+\mathrm{f}$, also lyıng near the real axis in the 4th quadrant

(v1) Now we want to go to $\varphi_{2} \varphi_{2}$ will have contributions from reggeons $\pi, \rho$, $A_{2}, \omega, f$ The isovectors, with large flip couplings, will dominate so we concentrate on those, the $\omega+f$ just go along with $\rho+A_{2}$ The full amplitude $\varphi_{2}$ is (as is known from np $\rightarrow$ pn) the pole contributions which must vanish at $t=0$, minus the absorptive correction which need not vanish at $t=0$

From spin properties, if the poles for $\rho+A_{2}$ lic in the 4th quadrant in $\varphi_{1}$ for - $t$ near 0 they must lie in the 2 nd quadrant for $\varphi_{2}$ for $t$ near zero, there is a relative minus sign from the spin states The phase of $\varphi_{2}$ at $t=0$ is that of the absorption correction, opposite to that of the pole, so the net $\rho+\mathrm{A}_{2}$ contribution to $\varphi_{2}$ at $t=0$ must be near the positive real axis

(vi1) Next the relative phase of $\pi$ and $\rho+\Lambda_{2}$ muxt be fixed Both are expected to be mainly real It is well established by analysis of data in both photoproduction [8] and in $n p \rightarrow$ pn [4], where the amplitude structures are equivalent, that the sharp small $t$ peak has $\pi$ and $\Lambda_{2}$ interfering constructively Thus $\pi$ will add to $\rho+A_{2}$ here, giving $\varphi_{2}$ at $t=0$ mainly real and positive

(vi11) Finally we must find the sign of $\operatorname{Im} \varphi_{2}$ at $t=0$ It arises from absorption since the poles vanish at $t=0$ We note, in two steps, that (a) absorbing a rotating pole with a purely imaginary pomeron gives a positive real to imaginary part ratio, and (b) absorbing with the pomeron phase taken into account (Re $P$ / Im $P$ negative over most of the $t$ range) goes the same direction, giving a positive ratio for $\varphi_{2}$ More explicitly,

$$
\varphi_{2}(s, t) \sim R_{2}(s, t)+\int b \mathrm{~d} b J_{0}(b \sqrt{-t}) R_{2}(s, b)[\operatorname{Im} P(s, b) \cdot \imath \operatorname{Re} P(s, b)],
$$

where $R_{2}$ is the reggeon pole, essentially real and negative for $t \sim 0, \operatorname{Im} P$ is the pomeron imaginary part, negative here by convention (if it were positive there would be a minus sign in front of the integral), and $\operatorname{Re} P$ is the pomeron real part, mainly positive Thus, the relative negative sign of $\operatorname{Re} P$ and $\operatorname{Im} P$ is cancelled by the $(-1)$ in front of $\operatorname{Re} P$ and so $\operatorname{Re} \varphi_{2}(s, 0) / \operatorname{Im} \varphi_{2}(s, 0)$ is positive $\varphi_{2}(0)$ is in the first quadrant.

(IX) Thus we find $\operatorname{Im} \varphi_{2}(0) / \operatorname{Im} \varphi_{1}(0)$ is negative Eqs (8) and (9) give (using $\left.\varphi_{1}=\varphi_{3}\right)$

$$
\frac{\Delta \sigma_{\mathrm{T}}}{\sigma_{\mathrm{T}}}=\frac{\sigma_{\Gamma}(\uparrow \uparrow)-\sigma_{\mathrm{T}}(\uparrow \downarrow)}{\sigma_{\mathrm{T}}}-\frac{\operatorname{Im} \varphi_{2}(0)}{\operatorname{Im} \varphi_{1}(0)}
$$

and so our argument gives a negative value for $\Delta \sigma_{\mathrm{T}}$

This is not the sign favored by the data at present [9], but the experimental error bars are still large

Although the argument is rather long* each step above is rather well founded in elther general terms or in terms of the behavior of other reactions If one (or

* If there is a simpler way to interpret $\operatorname{Im} \varphi_{2}(0)$, particularly from an exchange mechantsm picture, or to deduce the expected sign, we would be glad to learn it 
an odd number) of steps is incorrect it will be instructive to learn which Step (1) is perhaps most likely, followed by (8)

(x) To get the magnitude of $\operatorname{Im} \varphi_{2}(0)$ we first note that it is effectively bounded by the $n p \rightarrow$ pn data If all the $V+T$ contribution were isovector, which should be acceptable to $50 \%$ or so, then at $t=0$

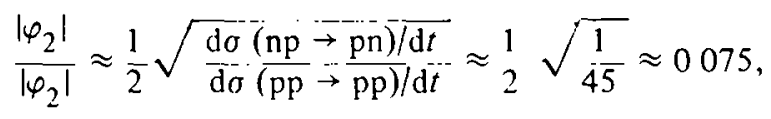

where the $\frac{1}{2}$ is an isopin factor Thus

$$
\left|\Delta \sigma_{\mathrm{T}}\right| \leqslant 0075 \sigma_{\mathrm{T}} \approx 3 \mathrm{mb}
$$

But $\varphi_{2}$ is mainly real and we really need $\operatorname{Im} \varphi_{2}(0)$, so we expect a smaller result As noted before, pole contributions give zero. The absorption model calculation of ref [4] predicts

$$
\Delta \sigma_{\mathrm{T}}=.18 \mathrm{mb} \text {, }
$$

it arrives at the sign obtained by our chain or arguments above since it is consistent with all the assumptions.

\section{$43 \Delta(\mathrm{d} \sigma / \mathrm{d} t)$}

From eq (23), the new quantity measured by polarized differential cross sections is

$$
2\left|\varphi_{5}\right|^{2}+\operatorname{Re}\left(\varphi_{1} \varphi_{2}^{*}-\varphi_{3} \varphi_{4}^{*}\right) \equiv \Delta(\mathrm{d} \sigma / \mathrm{d} t) 16 \pi q^{2} s
$$

Very little of a general nature can be said about $\Delta$ At $t=0, \varphi_{5}=\varphi_{4}=0$ so

$$
\begin{aligned}
& \Delta(\mathrm{d} \sigma / \mathrm{d} t)_{t=0}=+\operatorname{Re}\left(\varphi_{1} \varphi_{2}^{*}\right) \\
& \quad \approx+\operatorname{Re}\left[\left(l \operatorname{Im} \varphi_{1}\right)\left(\operatorname{Re} \varphi_{2}+\imath \operatorname{Im} \varphi_{2}\right)^{*}\right] \\
& \quad \approx+\operatorname{Im} \varphi_{1} \operatorname{Im} \varphi_{2},
\end{aligned}
$$

which is negative according to the steps above [e g step (ix)] Since $2\left|\varphi_{5}\right|^{2}$ is positive definite, if $\operatorname{Re}\left(\varphi_{1} \varphi_{2}^{*}-\varphi_{3} \varphi_{4}^{*}\right)$ ever gets small $\Delta(\mathrm{d} \sigma / \mathrm{d} t)$ will become positive The actual behavior of $\operatorname{Re}\left(\varphi_{1} \varphi_{2}^{*}-\varphi_{3} \varphi_{4}^{*}\right)$ depends sensitively on interference phases*

The model of ref [4] gives explicitly the curve in fig 1 The negative value at $t=0$ and the positive excursion can be seen It should be emphasized that the $t=0$ value is closely related to the result for $\Delta \sigma_{\mathrm{T}}$ If $\Delta \sigma_{\mathrm{T}}$ is positive the $4(\mathrm{~d} \sigma / \mathrm{d} t)$ at $t=0$ should be positive unless $\left|\operatorname{Re} \varphi_{1} \operatorname{Re} \varphi_{2}\right|>\left|\operatorname{Im} \varphi_{1} \operatorname{Im} \varphi_{2}\right|$,

* We have not found any simple interpretation of $\Delta(\mathrm{d} \sigma / \mathrm{d} t)$ 


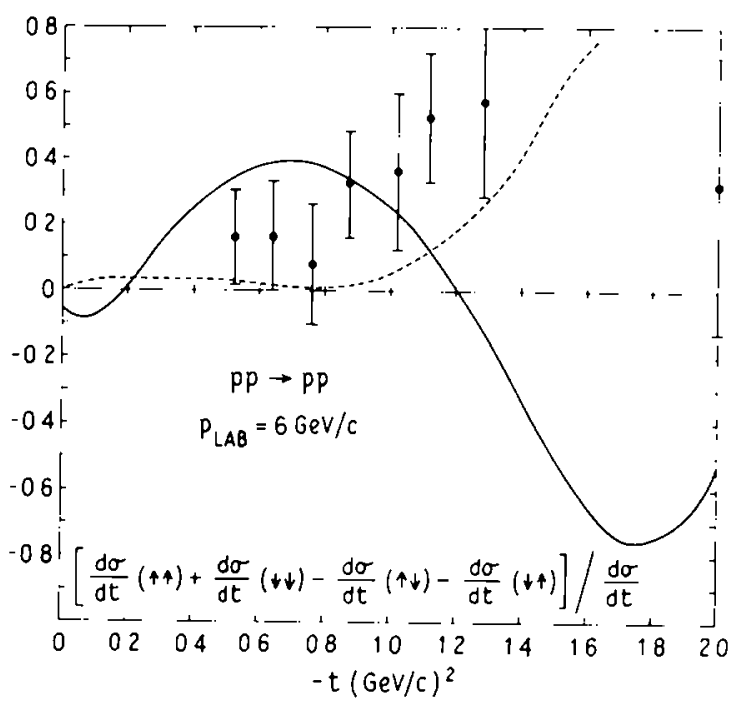

Fig 1. A plot of the measured quantity

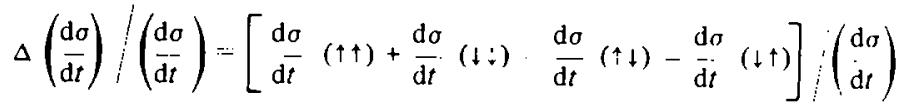

$$
\begin{aligned}
& \left.=8\left[2\left|\varphi_{5}\right|^{2}+\operatorname{Re}\left(\varphi_{1} \varphi_{2}^{*}-\varphi_{3} \varphi_{4}^{*}\right)\right] /|| \varphi_{1} i^{2}+\left|\varphi_{2}\right|^{2}+\left|\varphi_{3}\right|^{2}+\left.\left|\varphi_{4}{ }^{2}+4\right| \varphi_{5}\right|^{2}\right]
\end{aligned}
$$

as a function of $t$ at $P_{\mathrm{bb}}=6 \mathrm{GeV} / c$ This quantity is also often denoted by the Wolfenstein symbol $4 C_{n n}$ The solid curve is drawn from the numbers given in the specific model of ref [4] The dashed line corresponds to taking $\operatorname{Re}\left(\varphi_{1} \varphi_{2}^{*} \cdots \varphi_{3} \varphi_{4}^{*}\right)=0$ to see the effect of the separate pieces The data points are taken from O'Fallon et al, [10]

which is not expected In any case, knowing both $\Delta \sigma_{\mathrm{T}}$, and $\Delta(\mathrm{d} \sigma / \mathrm{d} t)$ near $t=0$ will be rather useful information, as discussed above at $t=0$ one can actually determine the amplitudes.

At larger $-t$ the behavior of $\Delta(\mathrm{d} \sigma / \mathrm{d} t)$ could be mapped out in terms of the amplitudes, but no point of qualitative significance appear to arise, and we content ourselves with fig 1 Perhaps from some other point of view a simpler interpretation will arise.

\section{References}

[1] L. Wolfenstein, Phys Rev 96 (1954) 1654, C R Schumacher and II A Bethe, Phys Rev 121 (1961) 1534, Proc of ANL Workshop on polanzed targets and beams, vol III, ANL/HEP-7208 $(1971)^{\dagger}$

+ To our knowledge, the results expressed in terms of the familar $s$-channel helicity ampltudes are not avallable in the literature 
[2] N H Buttımore, Cavendish Laboratory report, 1971, unpublıshed

[3] M Ross, F Henyey and G L Kane, Nucl Phys B13 (1970) 269

[4] G L Kane and $M$ Vaughn, in preparation

[5] H Pilkuhn, The interactions of hadrons, (North-Holland, Amsterdam, 1967)

[6] A B Wicklund, ANL preprint, private communication

[7] L Stodolsky and J.J Sakura1, Phys Rev Letters 11 (1963) 90

[8] R Worden, Nucl Phys B37 (1972) 253

19] E F Parker et al, Phys Rev Letters 31 (1973) 783

[10] J R O'Fallon et al, Phys Rev Letters 32 (1974) 77 